\title{
Novel tumor-targeting, self-assembling peptide nanofiber as a carrier for effective curcumin delivery
}

This article was published in the following Dove Press journal:

International Journal of Nanomedicine

24 December 2013

Number of times this article has been viewed

\author{
Jianfeng Liu \\ Jinjian Liu \\ Hongyan Xu \\ Yumin Zhang \\ Liping Chu \\ Qingfen Liu \\ Naling Song \\ Cuihong Yang
}

Tianjin Key Laboratory of Molecular Nuclear Medicine, Institute of Radiation Medicine, Chinese Academy of Medical Science and Peking Union Medical College, People's Republic of China
Correspondence: Cuihong Yang Institute of Radiation Medicine,

Chinese Academy of Medical Science,

Tianjin 30007I, People's Republic of China

Tel +862285682399

Fax +862285683033

Email yangcuihong1983@163.com

\begin{abstract}
The poor aqueous solubility and low bioavailability of curcumin restrict its clinical application for cancer treatment. In this study, a novel tumor-targeting nanofiber carrier was developed to improve the solubility and tumor-targeting ability of curcumin using a selfassembled Nap-GFFYG-RGD peptide. The morphologies of the peptide nanofiber and the curcumin-encapsulated nanofiber were visualized by transmission electron microscopy. The tumor-targeting activity of the curcumin-encapsulated Nap-GFFYG-RGD peptide nanofiber (f-RGD-Cur) was studied in vitro and in vivo, using Nap-GFFYG-RGE peptide nanofiber (f-RGE-Cur) as the control. Curcumin was encapsulated into the peptide nanofiber, which had a diameter of approximately 10-20 nm. Curcumin showed sustained-release behavior from the nanofibers in vitro. f-RGD-Cur showed much higher cellular uptake in $\alpha v \beta 3$ integrin-positive HepG2 liver carcinoma cells than did non-targeted f-RGE-Cur, thereby leading to significantly higher cytotoxicity. Ex vivo studies further demonstrated that curcumin could accumulate markedly in mouse tumors after administration of f-RGD-Cur via the tail vein. These results indicate that Nap-GFFYG-RGD peptide self-assembled nanofibers are a promising hydrophobic drug delivery system for targeted treatment of cancer.
\end{abstract}

Keywords: nanofiber, tumor-targeting, self-assembling, curcumin, drug delivery

\section{Introduction}

Because almost all chemotherapeutic antitumor agents have no tumor-targeting activity and cause serious adverse effects, their clinical application is restricted. Many nanomaterials have been proposed as drug carriers to overcome this barrier to the use of chemotherapeutic drugs. ${ }^{1}$ The enhanced permeability and retention effect of nanomaterials can improve the accumulation of chemotherapeutic agents at tumor sites. For example, liposomes, carbon nanotubes, dendrimers, and micelles have been used as carriers for SN38, doxorubicin, paclitaxel, and cisplatin to improve drug concentrations in tumors and reduce adverse effects. ${ }^{2-5}$ Another advantage of using nanomaterials as drug carriers is the enhanced solubility of chemotherapeutic drugs. Many of these agents have poor aqueous solubility, leading to low bioavailability. For example, the aqueous solubility of paclitaxel is $30 \mu \mathrm{g} / \mathrm{mL}$ and for hydroxycamptothecin is only $3.9 \mu \mathrm{g} / \mathrm{mL}$. The hydrophobic region of amphiphilic nanomaterials can solubilize drugs, and their hydrophilic region makes drugs soluble in an aqueous environment. ${ }^{6}$

Self-assembling peptide nanofibers have attracted considerable interest because of their good biocompatibility, easy modification, and design flexibility through a "bottom-up" approach. They have been widely used in three-dimensional cell culture, tissue engineering, and regenerative medicine research. ${ }^{89}$ Self-assembling peptide 
nanofibers have also been used as drug delivery systems to enhance solubility of a hydrophobic drug, improve accumulation at the tumor site, and reduce adverse effects. ${ }^{10}$ Hydrophobic antitumor drugs such as paclitaxel, ${ }^{11}$ camptothecin, ${ }^{12}$ and ellipticine ${ }^{13}$ encapsulated into peptide nanofibers have shown improved antitumor effects. Some studies have demonstrated that the two-dimensional structure of peptide nanofibers is superior to the three-dimensional structure of nanoparticles for drug carriers. For example, Law et al found that peptide-based nanofibers have better biocompatibility, better tumor targeting within a shorter time frame, and more rapid elimination compared with spherical nanomaterials (poly[lactic-co-glycolic acid], gold, polystyrene, cadmium and selenium quantum dots), and carbon rods. ${ }^{14}$

The conjugation of tumor-targeting agents to nanomaterials is another strategy for improving tumor accumulation and bioavailability of drugs. Peptides, ${ }^{15}$ proteins, ${ }^{16}$ aptamers, ${ }^{17}$ vitamins,${ }^{18}$ and polysaccharides ${ }^{19}$ have been developed as tumor-targeting agents. Among these molecules, the tripeptide motif, arginine-glycine-aspartic acid (RGD), has attracted great attention. RGD is the binding motif of integrin $\alpha v \beta 3$, which is overexpressed on the angiogenic endothelium in malignant tumors and is vital for the adhesion, signaling, migration, and survival of integrin $\alpha v \beta 3$ over-expressed cancer cells. ${ }^{20,21}$ It has been widely used for tumor diagnosis and targeting therapy. Liposomes, ${ }^{22}$ micelles, ${ }^{23}$ dendrimers ${ }^{24}$ and silica nanoparticles ${ }^{25}$ have been conjugated with RGD to introduce tumor-targeting activity. RGD has also been used in positron emission tomography, ${ }^{26}$ single photon emission computed tomography, ${ }^{27}$ magnetic resonance imaging, ${ }^{28}$ and optical ${ }^{29}$ imaging for diagnosis of cancer.

In this study, we report the design, synthesis, and characterization of a novel peptide nanofiber, self-assembled from Nap-GFFYG-RGD, as a tumor-targeting drug carrier. In order to study the RGD tumor-targeting activity, nanofibers self-assembled from Nap-GFFYG-RGE were also synthesized as the control. Curcumin, a hydrophobic antitumor agent, was used as the model drug. The nanofibers were characterized with respect to their morphology, drug encapsulation efficiency, and in vitro drug release. In vitro cytotoxicity was analyzed in human hepatocellular liver carcinoma (HepG2) and mouse embryonic fibroblast (NIH3T3) cell lines. Cellular uptake was investigated in HepG2, human embryonic kidney (293T), and human breast cancer (MCF-7) cell lines. Ex vivo biodistribution on HepG2 xenografts in nude mice was also studied to confirm the tumor-targeting activity of the nanofibers.

\section{Materials and methods}

Curcumin (diferuloyl methane) and peptides were purchased from Sigma-Aldrich Corporation (Carlsbad, CA, USA). 2-chlorotrityl chloride resin and Fmoc-amino acids were obtained from GL Biochem (Shanghai, People's Republic of China). 3-(4,5-dimethylthiazol-2-yl)-2,5-diphenyl tetrazolium bromide (MTT), dimethyl sulfoxide, and other chemical reagents and solvents were sourced from Alfa (Shenzhen, People's Republic of China). A dialysis membrane (molecular weight cut-off 1,000 Da) was purchased from BBI Inc (Shanghai, People's Republic of China). All cell culture media and supplies were purchased from Gibco Corporation (Grand Island, NY, USA).

NIH3T3, HepG2, 293T, and MCF-7cells were cultured in Roswell Park Memorial Institute 1640 medium supplemented with $10 \%$ fetal bovine serum, $100 \mathrm{U} / \mathrm{mL}$ penicillin, and $100 \mu \mathrm{g} / \mathrm{mL}$ streptomycin. The cells were maintained at $37^{\circ} \mathrm{C}$ with $5 \% \mathrm{CO}_{2}$. Other reagents were commercially available and used as received.

${ }^{1} \mathrm{H}$ nuclear magnetic resonance (NMR, Varian Unity Plus $400 \mathrm{mHz}$, Varian Instruments, Palo Alto, CA, USA) was used to characterize the peptides. Electrospray ionization tandem mass spectrometric analyses were performed on an LCQ AD system (Thermo Finnigan, Silicon Valley, CA, USA). Transmission electron microscopy was performed on a Tecnai G2 F20 system (FEI Co, Columbia, MD, USA) operating at $200 \mathrm{kV}$. The crude peptide was purified on a high-pressure liquid chromatography system (LumTech, Moorestown, Germany) using a $\mathrm{C} 18$ reversed phase column with $\mathrm{MeOH}$ $(0.1 \%$ trifluoroacetic acid) and water $(0.1 \%$ trifluoroacetic acid) as the eluents.

\section{Preparation and characterization of peptides}

The peptides, ie, Nap-GFFYG-RGD (p-RGD) and NapGFFYG-RGE (p-RGE), were prepared by solid phase peptide synthesis using 2-chlorotrityl chloride resin. The first Fmoc-protected amino acid was loaded onto the resin at the C-terminal. The Fmoc group of the amino acid was deprotected with $20 \%$ piperidine in anhydrous N,N'-dimethylformamide. The next Fmoc-protected amino acid was activated by N,Ndiisopropylethylamine and coupled to the free amino group

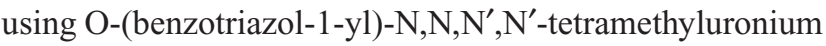
hexafluorophosphate as the coupling reagent. The growth of the peptide chain was allowed to proceed according to the standard Fmoc solid phase peptide synthesis protocol. After the last coupling step, the peptide derivatives were cleaved from the resin with a mixture of $1 \%$ trifluoroacetic acid and 
$99 \%$ dichloromethane for 10 minutes, then dried via rotary evaporation followed by diethylether precipitation. The crude products were purified by reverse phase high-pressure liquid chromatography. ${ }^{1} \mathrm{H}$ NMR spectroscopy and high-resolution mass spectrometry were used to characterize the peptides.

\section{Preparation of $f-R G D-C u r$ and $f-R G E-C u r$}

Fifty milligrams of curcumin were dissolved in $5 \mathrm{~mL}$ of anhydrous dimethyl sulfoxide and stirred at $40^{\circ} \mathrm{C}$. p-RGD and p-RGE were dissolved in phosphate-buffered saline ( $\mathrm{pH} 7.4)$ and the concentrations were adjusted to $3 \mathrm{mg} / \mathrm{mL}$. Next, $500 \mu \mathrm{L}$ of $\mathrm{p}-\mathrm{RGD}$ and $\mathrm{p}-\mathrm{RGE}$ solutions were heated to boiling point and cooled to $40^{\circ} \mathrm{C}$, and $22 \mu \mathrm{L}$ of curcumin solution at $40^{\circ} \mathrm{C}$ was then added to the $\mathrm{p}-\mathrm{RGD}$ and $\mathrm{p}-\mathrm{RGE}$ solutions. The reaction mixtures were incubated in a $40^{\circ} \mathrm{C}$ water bath for 2 hours. Curcumin-encapsulated Nap-GFFYG-RGD peptide nanofiber (f-RGD-Cur) and curcumin-encapsulated Nap-GFFYG-RGE peptide nanofiber (f-RGE-Cur) were then formed by incubation at room temperature for 2 hours. Unassembled curcumin was removed by centrifugation for 20 minutes at a speed of $1,500 \times \mathrm{g}$. The morphologies of the nanofibers were observed by transmission electron microscopy.

\section{Transmission electron microscopy}

Solutions of f-RGD, f-RGE, f-RGD-Cur, and f-RGE-Cur were prepared as described above. Next, $10 \mu \mathrm{L}$ samples of each were placed on a carbon-coated copper grid and incubated for 30 seconds to allow the peptide nanostructures to adhere to the substrate, then rinsed twice with ultrapure water. The samples were then stained with a saturated uranyl acetate solution and placed in a desiccator overnight prior to analysis.

\section{In vitro drug release}

In vitro drug release was evaluated using the dialysis method. Dialysis bags (molecular weight cut-off 1,000 Da) containing $0.5 \mathrm{~mL}$ of f-RGD-Cur or f-RGE-Cur solution were immersed in a thermostatic gas bath containing $20 \mathrm{~mL}$ of release medium $(0.5 \%$ Tween 80 in phosphate-buffered saline, $\mathrm{pH} 7.4$ ) at $37^{\circ} \mathrm{C}$. At predefined intervals, $1 \mathrm{~mL}$ aliquots of receiving buffer were withdrawn and replaced with the same volume of release medium. The drug-release behavior of the carriers was determined by ultraviolet spectrophotometry (Agilent 8453, Agilent Technologies, Santa Barbara, CA, USA) at $428 \mathrm{~nm}$.

\section{In vitro cellular uptake}

Curcumin has an intrinsic fluorescence spectrum that is often used to monitor its localization. 293T, MCF-7, and HepG2 cells were seeded into 24 -well plates at a density of $3 \times 10^{4}$ cells/well in $500 \mu \mathrm{L}$ of Roswell Park Memorial Institute 1640 medium supplemented with $10 \%$ fetal bovine serum. After incubating for 24 hours, the culture medium in each well was replaced with $500 \mu \mathrm{L}$ of fresh medium containing $10 \mu \mathrm{g} / \mathrm{mL}$ of free curcumin or equivalent curcumin-containing f-RGD-Cur or f-RGE-Cur. After incubating for 4 hours, the cells were rinsed three times with $500 \mu \mathrm{L}$ of phosphate-buffered saline to remove the free curcumin, f-RGD-Cur, or f-RGE-Cur. Cellular uptake was visualized on an inverted fluorescence microscope (DMI6000B, Leica, Wetzlar, Germany).

\section{In vitro cytotoxicity}

The cytotoxicity of free curcumin, f-RGD-Cur, and f-RGECur was evaluated using the MTT assay with NIH3T3 and HepG2 cells. The cells were seeded into 96-well plates at a density of $1 \times 10^{4}$ cells/well in $100 \mu \mathrm{L}$ of culture medium and incubated for 24 hours. Next, $100 \mu \mathrm{L}$ of culture medium containing different concentrations of free curcumin, f-RGD-Cur, or f-RGE-Cur were added to 96-well plates for 24 hours. Each treatment was repeated in eight wells. After incubation for the specified time at $37^{\circ} \mathrm{C}$ in a humidified incubator, $20 \mu \mathrm{L}$ of MTT solution $(5 \mathrm{mg} / \mathrm{mL}$ in phosphatebuffered saline) were added to each well. Four hours later, the medium containing MTT was removed and $150 \mu \mathrm{L}$ of dimethyl sulfoxide were added to dissolve the formazan crystals. The optical density of the solution was measured at $570 \mathrm{~nm}$ on a microplate reader (Varioskan Flash, Thermo Fisher Scientific, South Logan, UT, USA). The effect of each agent on growth inhibition was determined as a percentage of the viability of cells treated with nanofiber without curcumin, for which the viability was taken as $100 \%$. The cell viability was calculated from the equation:

\section{Cell viability $(\%)=$ Average OD of test group/average OD of control group $\times 100 \%$.}

\section{Ex vivo distribution}

BALB/c mice (male, aged 4-6 weeks, weight 18-22 g) were purchased from the Laboratory Animal Center of The Academy of Military Medical Sciences (Beijing, People's Republic of China). All animal procedures were performed according to the guidelines of the Regulations for the Administration of Affairs Concerning Experimental Animals (Tianjin, People's Republic of China, revised in June 2004) and adhered to the Guiding Principles in the Care and Use of Animals of the American Physiological Society. HepG2 cells $\left(1 \times 10^{7}\right)$ suspended in phosphate-buffered saline were subcutaneously 
injected into the right flank to produce liver cancer xenografts. About 2 weeks after inoculation, when the tumors had reached a volume of approximately $300 \mathrm{~mm}^{3}$, the mice were randomized into three groups ( $\mathrm{n}=5 \mathrm{each})$, and $200 \mu \mathrm{L}(10 \mu \mathrm{g} / \mathrm{mL})$ of free curcumin or equivalent curcumin-containing f-RGD-Cur or f-RGE-Cur were injected via the tail vein. Four hours following injection, the mice were sacrificed, and the heart, liver, spleen, lung, kidneys, and tumor were isolated under white light and immediately scanned using a Kodak IS in vivo FX imaging system (Kodak, New Haven, CT, USA). The fluorescence images were acquired using a $464 \mathrm{~nm}$ excitation filter and $535 \mathrm{~nm}$ emission filter. The relative fluorescence intensity per unit area was calculated using Kodak 5.1 software.

\section{Statistical analysis}

All statistical analysis was performed using Statistical Package for the Social Sciences version 16 software (SPSS Inc, Chicago, IL, USA). The statistical significance of differences was determined by one-way analysis of variance. All statistical tests were two-sided, and $P$-values less than 0.05 were considered to be statistically significant. The results are shown as mean \pm standard deviation.

\section{Results and discussion \\ Preparation and characterization of $f-R G D-C u r$ and $f-R G E-C u r$}

Recent studies have indicated that the shape of nanostructures may greatly affect their in vivo biodistribution, and some flexible filament nanostructures have shown longer blood circulation and better antitumor effects than spherical nanostructures. ${ }^{14,30,31}$ The p-RGD and p-RGE peptides were synthesized (Figures 1A and S1-S4) and used to form nanofiber skeletons by self-assembly via electrostatic and hydrophobic interactions. The peptides formed single-layer nanofibers (approximately 10-20 nm in width) that could be visualized by transmission electron microscopy (Figure 2A and B).

A

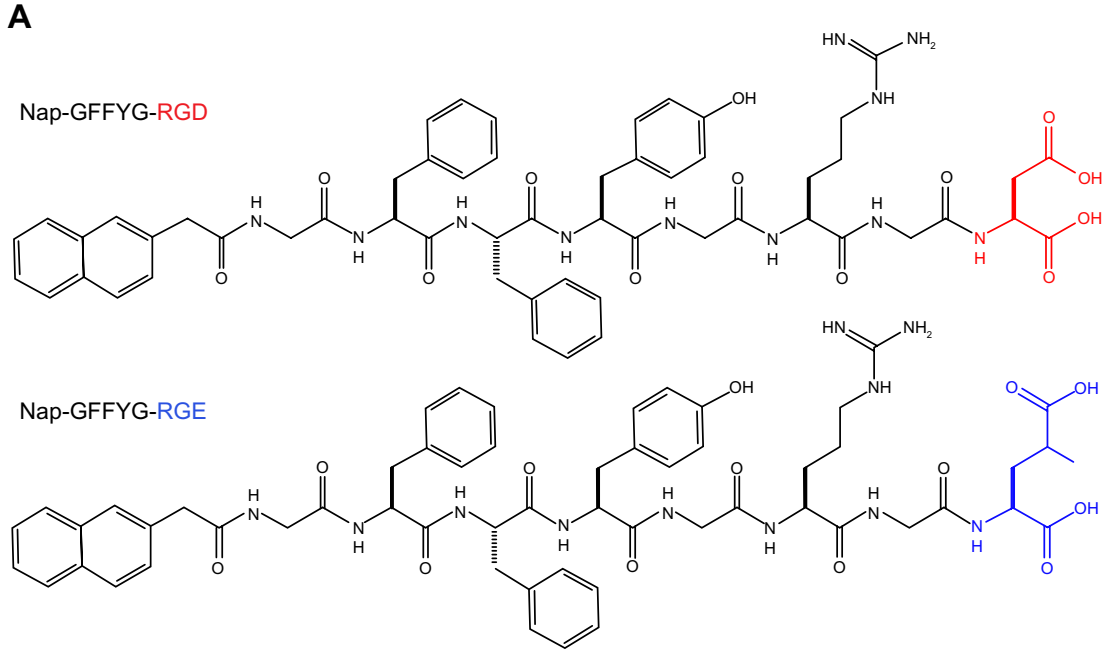

B

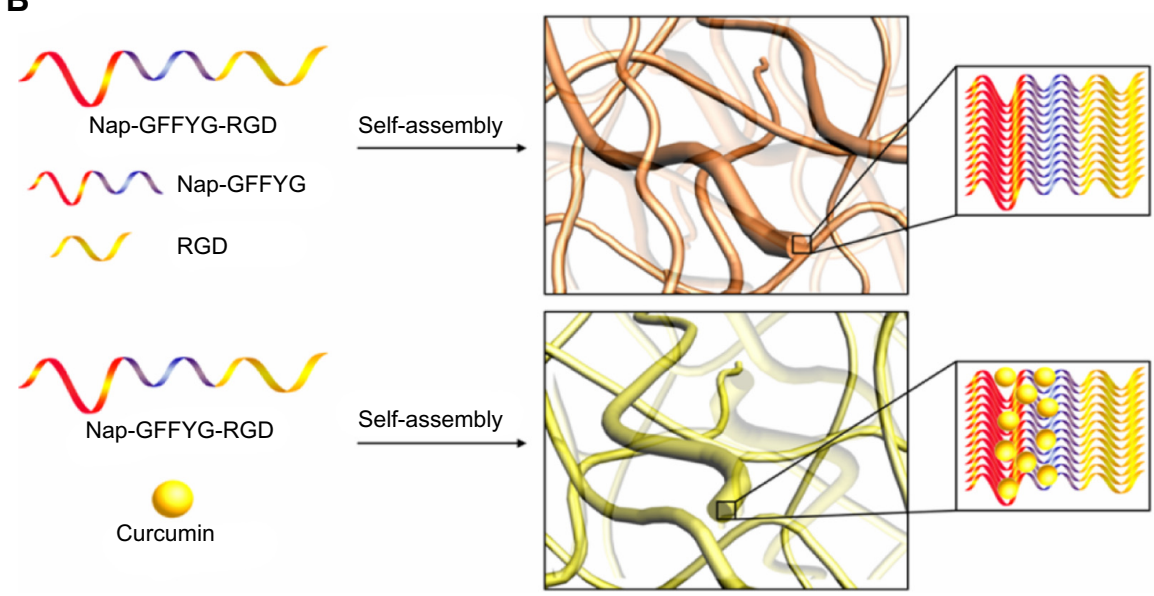

Figure I (A) Molecular structures of Nap-GFFYG-RGD and Nap-GFFYG-RGE. (B) Schematic illustration for formation of Nap-GFFYG-RGD peptide nanofibers and encapsulation of curcumin. 


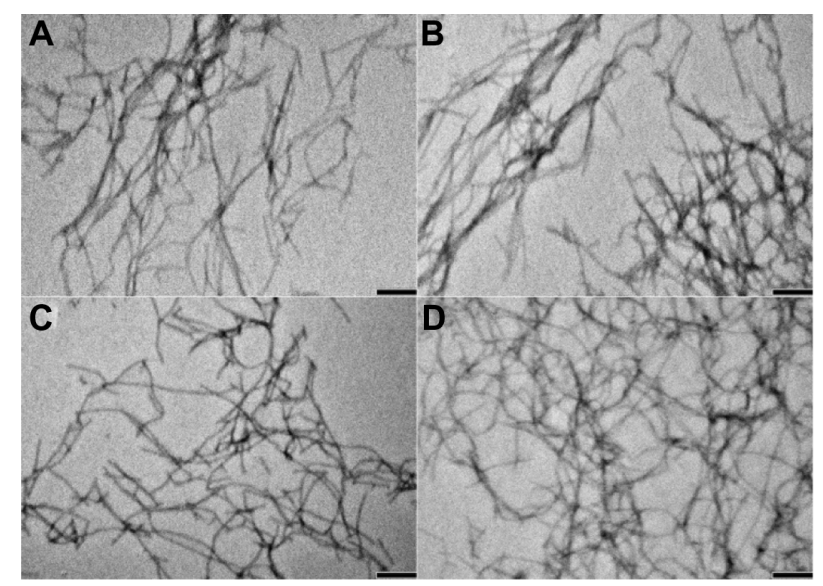

Figure 2 Transmission electron microscopic images of peptide nanofibers. (A) f-RGD, (B) f-RGE, and curcumin-encapsulated nanofibers (C) f-RGD-Cur and (D) f-RGD-Cur. Scale bar, $200 \mathrm{~nm}$. The peptides could self-assemble into nanofibers (approximately 10-20 nm in width). Curcumin could be encapsulated into the nanofibers without any obvious effects on the shape of the nanofibers.

Abbreviations: f-RGD-Cur, curcumin-encapsulated Nap-GFFYG-RGD peptide nanofiber; f-RGE-Cur, curcumin-encapsulated Nap-GFFYG-RGE peptide nanofiber.

Curcumin was encapsulated into the nanofibers without any obvious effects on the shape of the nanofibers (Figure 2C and D). This result is consistent with the report of Zhang et al. ${ }^{11}$ Figure $1 \mathrm{~B}$ shows the Nap-GFFYG-RGD peptide selfassembling into nanofibers and drug encapsulation.

\section{Encapsulation efficiency and in vitro release of curcumin}

As a natural medicine used in Asia, curcumin extracted from different plants has been shown to have potential antitumor activity. It can suppress the expression of epidermal growth factor receptor and estrogen receptors, which are cancer-associated growth factor receptors. ${ }^{32}$ Curcumin has been widely used in antitumor research, but its poor solubility in water results in poor bioavailability and restricts its clinical application. ${ }^{33,34}$ Amphiphilic nanomaterials have been used to improve the solubility of curcumin. ${ }^{35,36}$ Zhang et al found that the hydrophobic drug, paclitaxel, could be encapsulated into the hydrophobic core formed by self-assembled peptide nanofibers. ${ }^{11}$ Pochan et al also reported that curcumin could interact with hydrophobic peptide moieties and encapsulate into self-assembled peptide hydrogels. ${ }^{37}$ Figure 3A shows the encapsulation efficiency of curcumin with RGD and RGE peptide nanofibers. The amount of curcumin solubilized in the nanofibers increased with the peptide concentration from $0.5 \mathrm{mg} / \mathrm{mL}$ to $5 \mathrm{mg} / \mathrm{mL}$. When the concentration of curcumin was $0.5 \mathrm{mM}$ and the peptide concentration was $5 \mathrm{mg} / \mathrm{mL}$, the efficiency of encapsulation of curcumin into the nanofibers reached about $32 \%$.

Controlled and sustained drug release is a desirable feature of drug carriers. In order to verify the sustained drug release characteristics of curcumin-loaded peptide nanofibers, the in vitro release of f-RGD-Cur and f-RGE-Cur was studied using a dialysis method. The drug release profile was performed under simulated physiologic conditions ( $\mathrm{pH} 7.4$ ). As shown in Figure 3B, both f-RGD-Cur and f-RGE-Cur showed a sustained drug release profile, with total curcumin release from the nanofibers being about $51.6 \%$ and $60.9 \%$, respectively, while treatment with free curcumin led to release of more than $95 \%$ of the drug.

\section{In vitro cellular uptake}

One of the advantages of curcumin is its intrinsic fluorescence, which can be detected directly to quantify its cellular uptake and intracellular trafficking. We used fluorescence microscopy to compare the fluorescence intensity of free curcumin, f-RGD-Cur, and f-RGE-Cur in normal human 293 T cells, human MCF-7 cancer cells with low expression of $\alpha v \beta 3$, and human HepG2 cancer cells with high expression of $\alpha v \beta 3$. To better compare their internalization, parameters such as laser power, offset, sensitivity, and gain constant were
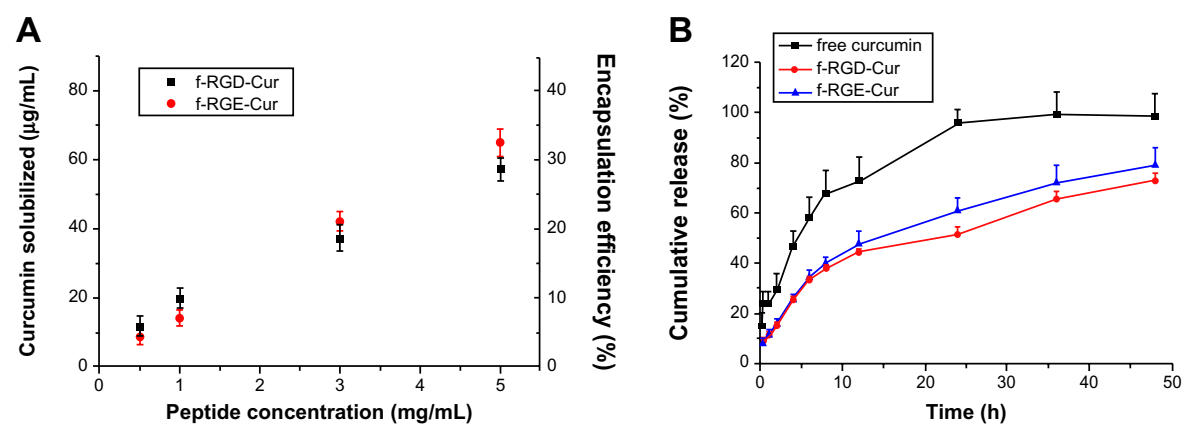

Figure 3 Encapsulation (A) and release profiles (B) of curcumin from f-RGD-Cur and f-RGE-Cur in release medium (0.5\% Tween 80 in phosphate-buffered saline, pH 7.4) at $37^{\circ} \mathrm{C}$. f-RGD-Cur and f-RGE-Cur had almost the same encapsulation efficiency and release properties. Both f-RGD-Cur and f-RGE-Cur showed a sustained drug release profile.

Abbreviations: f-RGD-Cur, curcumin-encapsulated Nap-GFFYG-RGD peptide nanofiber; f-RGE-Cur, curcumin-encapsulated Nap-GFFYG-RGE peptide nanofiber; h, hour. 


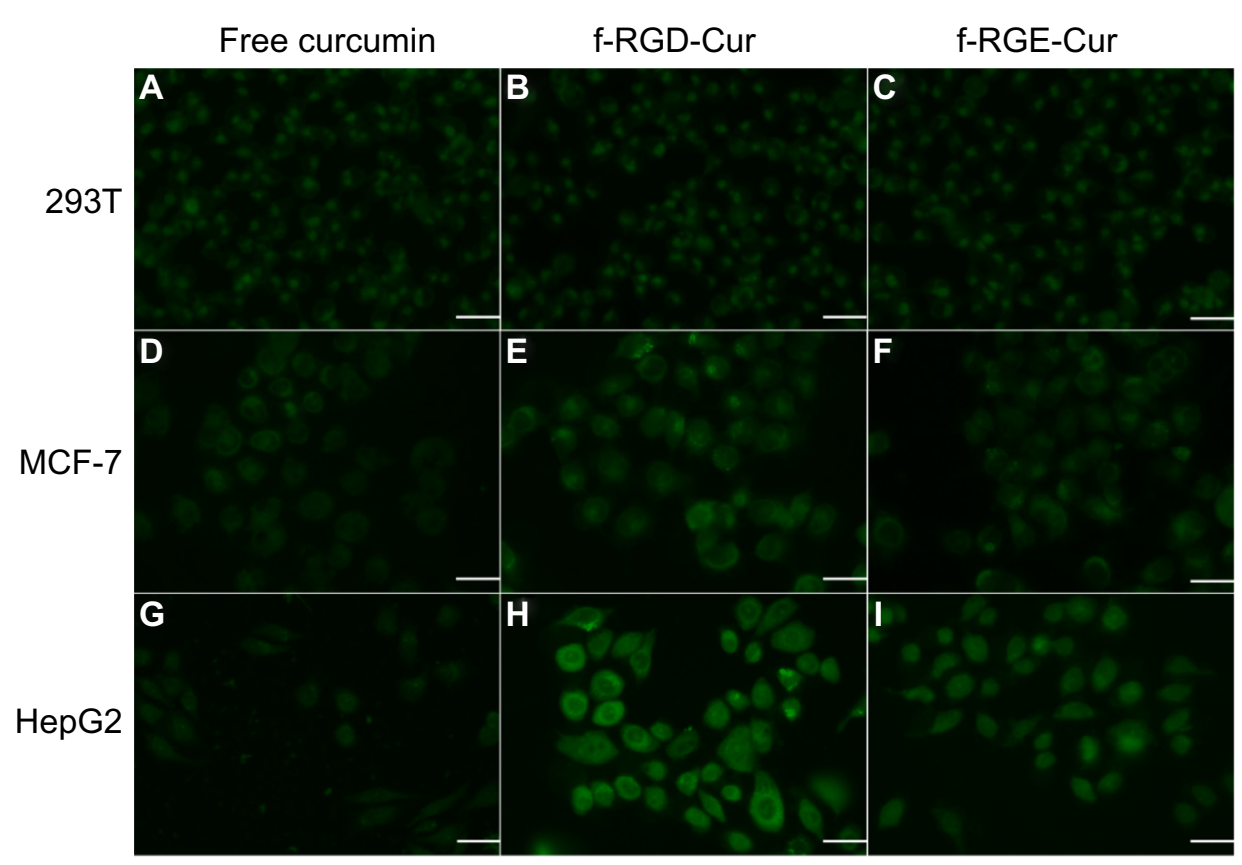

Figure 4 Uptake of various curcumin formulations by 293T (A-C), MCF-7 (D-F), and HepG2 (G-I) cells. The cells were incubated with free curcumin, f-RGD-Cur, or $\mathrm{f}$-RGE-Cur at an equivalent curcumin concentration of $10 \mu \mathrm{g} / \mathrm{mL}$ for 4 hours. Scale bar, $30 \mu \mathrm{m}$. Uptake of f-RGD-Cur was greater by HepG2 cells overexpressing integrin $\alpha v \beta 3$ than by $\alpha v \beta 3$-negtive MCF-7 cells and 293T cells.

Abbreviations: f-RGD-Cur, curcumin-encapsulated Nap-GFFYG-RGD peptide nanofiber; f-RGE-Cur, curcumin-encapsulated Nap-GFFYG-RGE peptide nanofiber.

harmonized during the cell imaging procedure. As shown in Figure 4, very similar and low fluorescence intensity was observed in normal human 293T cells incubated with free curcumin, f-RGD-Cur, or f-RGE-Cur (Figure 4A-C). For MCF-7 cells, the fluorescence intensity was slightly higher when they were incubated with f-RGD-Cur rather than with free curcumin or f-RGE-Cur (Figure 4D-F). In HepG2 cells, the fluorescence intensity following incubation with f-RGDCur was much higher than following incubation with free curcumin and f-RGE-Cur (Figure 4G-I). The cells incubated with free curcumin showed the lowest fluorescence intensity, and the fluorescence intensity was HepG2 > MCF-7 > 293T cells when incubated with f-RGD-Cur (Figure 4B, E, and H). These results indicate that cellular uptake of f-RGD-Cur was higher in cancer cells overexpressing integrin $\alpha v \beta 3$ than in $\alpha v \beta 3$-negtive cancer cells and normal cells and that the RGD-containing peptide self-assembled nanofibers possessed in vitro cancer cell-targeting activity.

\section{In vitro cytotoxicity}

The cytotoxicity of free curcumin, p-RGD, p-RGE, f-RGDCur, and f-RGE-Cur was evaluated by MTT assay in the HepG2 cell line overexpressing integrin $\alpha v \beta 3$, using the normal NIH3T3 cell line with low expression of integrin $\alpha v \beta 3$ as the control. The peptide nanofibers showed good biocompatibility, and the viability of both normal cells and tumor cells was greater than $80 \%$ when the peptide concentration was less than $180 \mu \mathrm{g} / \mathrm{mL}$ (Figure $5 \mathrm{~A}$ and C). f-RGD-Cur showed lower cytotoxicity than f-RGE-Cur in normal NIH3T3 cells (Figure 5B), but higher cytotoxicity in HepG2 tumor cells (Figure 5D). This may be caused by the active targeting ability of RGD, which could enable f-RGD-Cur to kill tumor cells more efficiently and thus protect normal tissues. Free curcumin showed higher cytotoxicity than f-RGD-Cur and f-RGE-Cur. The phenomenon of free drug showing higher cytotoxicity in normal cells than in cancer cells and lower cell viability than nanoparticle-encapsulated drugs has also been reported by $\mathrm{Xu}$ et $\mathrm{al}^{38}$ and $\mathrm{Su}$ et al, ${ }^{39}$ and may be caused by the controlled and sustained release of active drug from f-RGD-Cur and $\mathrm{f}-\mathrm{RGE}-\mathrm{Cur}$. From the in vitro release results, we found that free curcumin had higher drug release than f-RGD-Cur and f-RGE-Cur, and this may the reason for the higher cytotoxicity of free curcumin.

\section{Ex vivo distribution}

Because of the high proteolytic activity and low $\mathrm{pH}$ in the gastrointestinal tract, oral delivery of intact peptide nanofibers to the target site is difficult. The low permeability of peptides at the intestinal barrier is another factor adding 

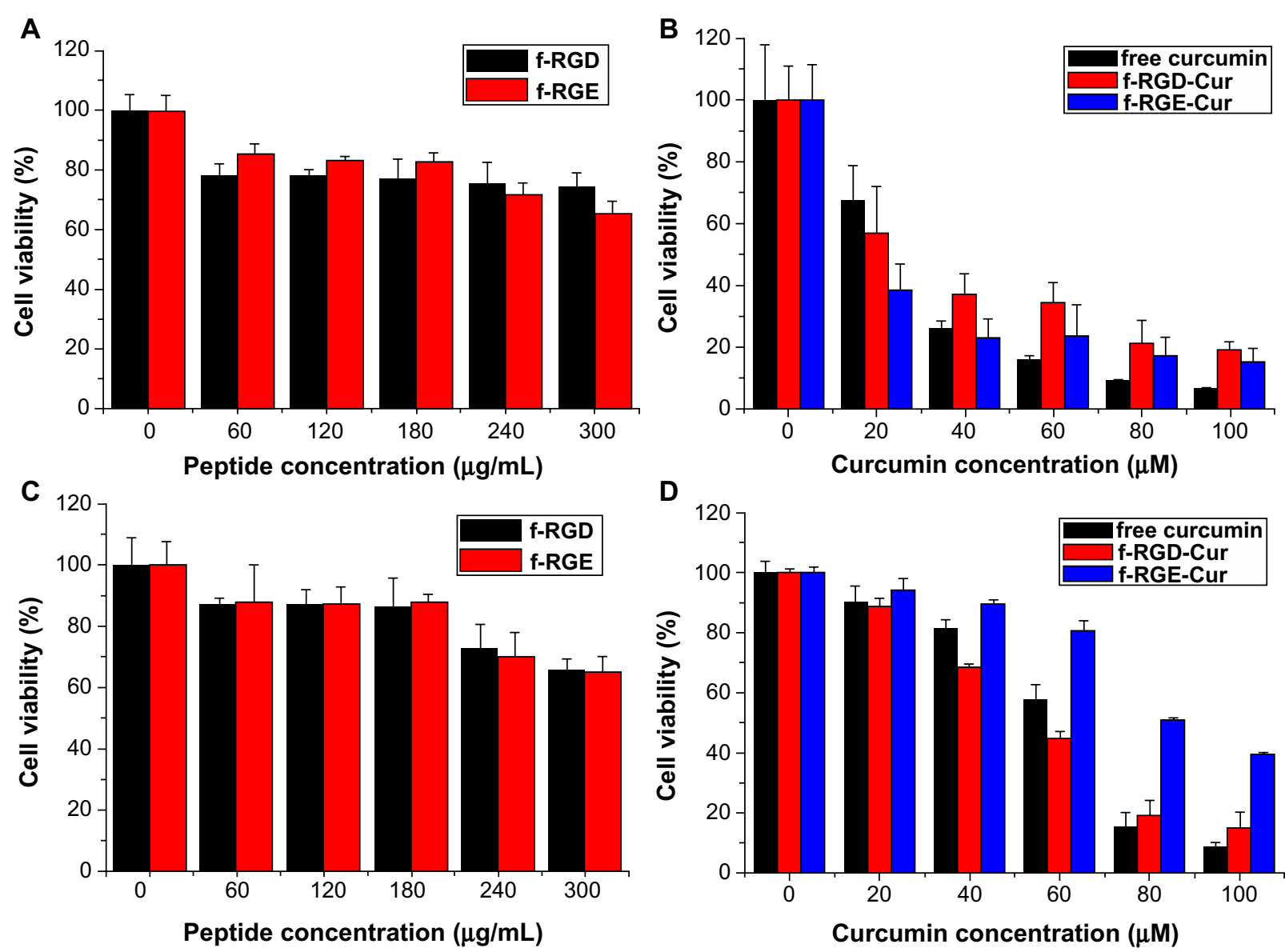

Figure 5 In vitro cytotoxicity of free curcumin, p-RGD, p-RGE, f-RGD-Cur, and f-RGE-Cur in NIH3T3 cells (A and B) and HepG2 cells (C and D) at 24 hours. p-RGD and p-RGE showed slight cytotoxicity to normal and cancer cells (A and C); f-RGD-Cur and f-RGE-Cur showed almost the same cytotoxicity to normal NIH3T3 cells (B), while f-RGD-Cur showed significantly greater cytotoxicity than f-RGE-Cur to HepG2 cells.

Abbreviations: f-RGD-Cur, curcumin-encapsulated Nap-GFFYG-RGD peptide nanofiber; f-RGE-Cur, curcumin-encapsulated Nap-GFFYG-RGE peptide nanofiber.

to their low bioavailability. ${ }^{40,41}$ Gastrointestinal delivery of peptide nanofibers is not feasible. Upon intravenous administration, nanostructures will undergo adsorption or opsonization by serum proteins, which results in a change in the physicochemical properties of the nanostructures. ${ }^{42}$ This may affect their half-life in blood and their biodistribution. Therefore, some agents show good targeting ability in vitro but no effect in vivo. Ex vivo optical imaging may have some diagnostic value because it is not subject to interference from overlying tissues, eg, skin and blood vessels, and thus closely resembles intraoperative procedures in surgical oncology as opposed to whole-body imaging. ${ }^{13}$ To determine whether curcumin accumulates efficiently at tumor sites after injection of f-RGD-Cur, ex vivo imaging of all major organs was performed 4 hours post-injection using a tumor xenograft assay. Representative color-coded fluorescence images of the excised tumors and tissues (heart, liver, spleen, lung, and kidney) obtained 4 hours post-treatment are shown in Figure 6A. These images show that the tumor group treated with f-RGD-Cur had the strongest curcumin fluorescence intensity, followed by tumors in the f-RGE-Cur group. Minimal curcumin fluorescence intensity was observed for tumors in the free curcumin group. The total fluorescence intensity in the free curcumin group was lower than in the f-RGD-Cur and f-RGE-Cur groups. As shown in Figure 6B, the fluorescence intensity in the f-RGDCur group was about 4-fold higher than that in the free curcumin group $(P<0.01)$. In addition, the fluorescence intensity in the f-RGD-Cur group was also significantly higher than in the f-RGE-Cur group $(P<0.05)$. Our results indicate that these peptide nanofibers can deliver curcumin into tumors, perhaps as a result of the enhanced permeability and retention effect. The RGD motif may enable RGD-containing peptide nanofibers to accumulate more effectively in tumors by active tumor-targeting. Further in 


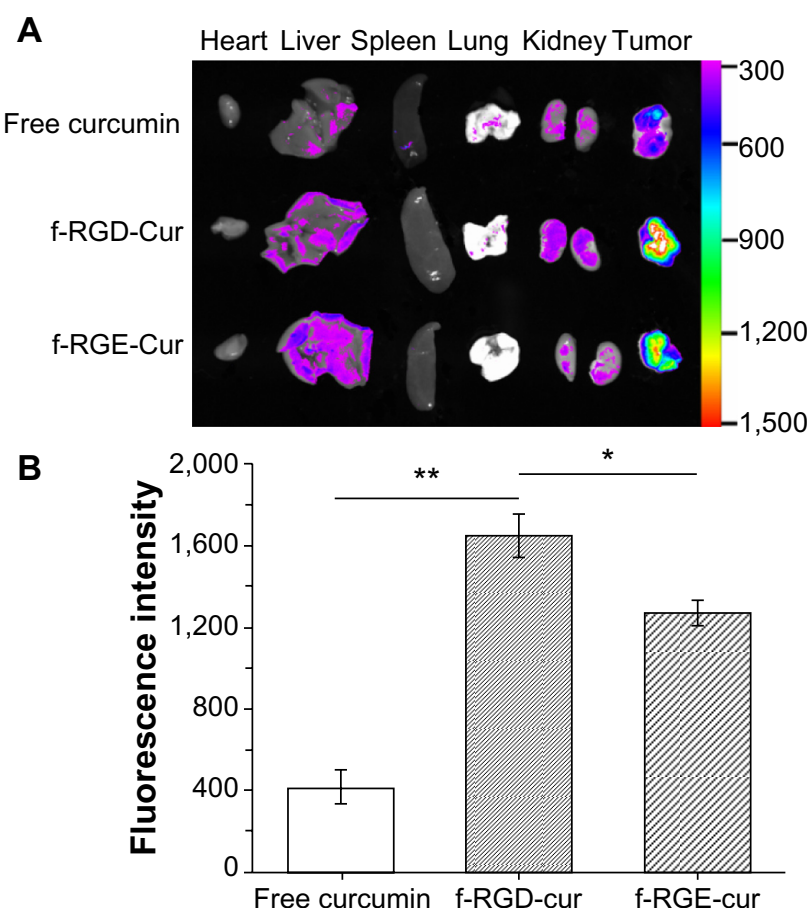

Figure 6 Ex vivo fluorescence images of tissues and tumors. Tissues were isolated from animals treated with $200 \mu \mathrm{L}(10 \mu \mathrm{g} / \mathrm{mL})$ of free curcumin or equivalent curcumin containing f-RGD-Cur or f-RGE-Cur4 hours after intravenous administration $(n=5)$. (A) Representative images from one of the experiments for the organs. The fluorescence intensity reflects the concentration of curcumin. f-RGD-Cur showed the highest accumulation in tumors, followed by f-RGE-Cur, and free curcumin had the lowest distribution in tumors. (B) Relative fluorescence intensity of curcumin in the excised tumors. ${ }^{*} \mathrm{P} P<0.01$ for $\mathrm{f}-\mathrm{RGD}$-Cur compared with the free curcumin group, indicating a very significant difference in curcumin concentration between the two groups; ${ }^{*} p<0.05$ for $\mathrm{f}-\mathrm{RGD}$-Cur versus the $\mathrm{f}$-RGE-Cur group, indicating a significant difference in curcumin concentration between the two groups.

Abbreviations: f-RGD-Cur, curcumin-encapsulated Nap-GFFYG-RGD peptide nanofiber; f-RGE-Cur, curcumin-encapsulated Nap-GFFYG-RGE peptide nanofiber.

vivo studies should be performed to determine the antitumor effects and toxicity of these nanofibers in targeted tumor therapy.

\section{Conclusion}

In this study, we have identified a new tumor-targeting peptide nanofiber, f-RGD-Cur, formed by the Nap-GFFYG peptide together with an RGD peptide as the tumor-targeting agent. This nanofiber efficiently encapsulated the hydrophobic drug, curcumin, and showed sustained drug release. Compared with free curcumin and non-targeting f-RGE-Cur nanofibers, f-RGD-Cur showed highly selective targeting of tumor cells and xenografts overexpressing integrin $\alpha v \beta 3$. f-RGD-Cur also demonstrated higher cytotoxicity than f-RGE-Cur to tumor cells and lower cytotoxicity to normal cells. These results suggest that RGD-containing peptide nanofibers may provide a useful platform for developing hydrophobic drug delivery carriers with tumor-targeting activity.

\section{Acknowledgments}

We thank the Science Foundation of China (81171371, 51203189), Tianjin Natural Science Foundation (13JCZDJC28100), PUMC Youth Fund and the Fundamental Research Funds for the Central Universities (3332013045), and the Development Foundation of the Institute of Radiation Medicine, Chinese Academy of Medical Sciences and Peking Union Medical College (SF1209, SZ1334) for financial support.

\section{Disclosure}

The authors report no conflicts of interest in this work.

\section{References}

1. Najer A, Wu D, Vasquez D, Palivan CG, Meier W. Polymer nanocompartments in broad-spectrum medical applications. Nanomedicine (Lond). 2013;8(3):425-447.

2. Bala V, Rao S, Boyd BJ, Prestidge CA. Prodrug and nanomedicine approaches for the delivery of the camptothecin analogue SN38. J Control Release. 2013;172(1):48-61.

3. Das M, Singh RP, Datir SR, Jain S. Intranuclear drug delivery and effective in vivo cancer therapy via estradiol-PEG-appended multiwalled carbon nanotubes. Mol Pharm. August 19, 2013. [Epub ahead of print.]

4. Teow HM, Zhou Z, Najlah M, Yusof SR, Abbott NJ, D'Emanuele A. Delivery of paclitaxel across cellular barriers using a dendrimer-based nanocarrier. Int J Pharm. 2013;441(1-2):701-711.

5. Qi X, Li N, Gu H, et al. Amphiphilic oligomer-based micelles as cisplatin nanocarriers for cancer therapy. Nanoscale. 2013;5(19):8925-8929.

6. Jing X, Deng L, Gao B, et al. A novel polyethylene glycol mediated lipid nanoemulsion as drug delivery carrier for paclitaxel. Nanomedicine. August 20, 2013. [Epub ahead of print.]

7. Yang C, Li D, Liu Z, et al. Responsive small molecular hydrogels based on adamantane-peptides for cell culture. J Phys Chem B. 2012;116(1): 633-638.

8. Nune M, Kumaraswamy P, Krishnan UM, Sethuraman S. Selfassembling peptide nanofibrous scaffolds for tissue engineering: novel approaches and strategies for effective functional regeneration. Curr Protein Pept Sci. 2013;14(1):70-84.

9. Hu Y, Wang H, Wang J, et al. Supramolecular hydrogels inspired by collagen for tissue engineering. Org Biomol Chem. 2010;8(14):3267-3271.

10. Wang H, Wei J, Yang C, et al. The inhibition of tumor growth and metastasis by self-assembled nanofibers of Taxol. Biomaterials. 2012; 33(24):5848-5853.

11. Zhang P, Cheetham AG, Lin YA, Cui H. Self-assembled Tat nanofibers as effective drug carrier and transporter. ACS Nano. 2013;7(7):5965-5977.

12. Soukasene S, Toft DJ, Moyer TJ, et al. Antitumor activity of peptide amphiphile nanofiber-encapsulated camptothecin. ACS Nano. 2011;5(11):9113-9121.

13. Cho H, Indig GL, Weichert J, Shin HC, Kwon GS. In vivo cancer imaging by poly(ethylene glycol)-b-poly(varepsilon-caprolactone) micelles containing a near-infrared probe. Nanomedicine. 2012;8(2): 228-236.

14. Wagh A, Singh J, Qian S, Law B. A short circulating peptide nanofiber as a carrier for tumoral delivery. Nanomedicine. 2013;9(4):449-457.

15. Liu J, Liu J, Chu L, et al. Novel peptide-dendrimer conjugates as drug carriers for targeting nonsmall cell lung cancer. Int J Nanomedicine. 2011;6:59-69.

16. Kim TH, Jo YG, Jiang HH, et al. PEG-transferrin conjugated TRAIL (TNF-related apoptosis-inducing ligand) for therapeutic tumor targeting. $J$ Control Release. 2012;162(2):422-428. 
17. Li X, Zhao Q, Qiu L. Smart ligand: Aptamer-mediated targeted delivery of chemotherapeutic drugs and siRNA for cancer therapy. $J$ Control Release. 2013;171(2):152-162.

18. Li J, Zheng L, Cai H, et al. Polyethyleneimine-mediated synthesis of folic acid-targeted iron oxide nanoparticles for in vivo tumor MR imaging. Biomaterials. 2013;34(33):8382-8392.

19. Yu Z, Schmaltz RM, Bozeman TC, et al. Selective tumor cell targeting by the disaccharide moiety of bleomycin. JAm Chem Soc. 2013;135(8): 2883-2886.

20. Cabodi S, Di Stefano P, Leal MP, et al. Integrins and signal transduction. Adv Exp Med Biol. 2010;674:43-54.

21. Hynes RO. A reevaluation of integrins as regulators of angiogenesis. Nat Med. 2002;8(9):918-921.

22. Chen Z, Deng J, Zhao Y, Tao T. Cyclic RGD peptide-modified liposomal drug delivery system: enhanced cellular uptake in vitro and improved pharmacokinetics in rats. Int J Nanomedicine. 2012;7:3803-3811.

23. Cai LL, Liu P, Li X, et al. RGD peptide-mediated chitosan-based polymeric micelles targeting delivery for integrin-overexpressing tumor cells. Int J Nanomedicine. 2011;6:3499-3508.

24. Zhu S, Qian L, Hong M, Zhang L, Pei Y, Jiang Y. RGD-modified PEGPAMAM-DOX conjugate: in vitro and in vivo targeting to both tumor neovascular endothelial cells and tumor cells. Adv Mater. 2011;23(12): H84-H89.

25. Fang IJ, Slowing II, Wu KC, Lin VS, Trewyn BG. Ligand conformation dictates membrane and endosomal trafficking of arginine-glycineaspartate (RGD)-functionalized mesoporous silica nanoparticles. Chemistry. 2012;18(25):7787-7792.

26. Zhu L, Guo N, Li Q, et al. Dynamic PET and optical imaging and compartment modeling using a dual-labeled cyclic RGD peptide probe. Theranostics. 2012;2(8):746-756.

27. Shao G, Zhou Y, Wang F, Liu S. Monitoring glioma growth and tumor necrosis with the U-SPECT-II/CT scanner by targeting integrin $\alpha v \beta 3$. Mol Imaging. 2013;12(1):39-48.

28. Zheng S, Huang M, Hong R, et al. RGD-conjugated iron oxide magnetic nanoparticles for magnetic resonance imaging contrast enhancement and hyperthermia. J Biomater Appl. June 24, 2013. [Epub ahead of print.]

29. Lu Y, Zhong Y, Wang J, et al. Aqueous synthesized near-infraredemitting quantum dots for RGD-based in vivo active tumour targeting. Nanotechnology. 2013;24(13):135101

30. Geng Y, Dalhaimer P, Cai S, et al. Shape effects of filaments versus spherical particles in flow and drug delivery. Nat Nanotechnol. 2007; 2(4):249-255.

31. Christian DA, Cai S, Garbuzenko OB, et al. Flexible filaments for in vivo imaging and delivery: persistent circulation of filomicelles opens the dosage window for sustained tumor shrinkage. Mol Pharm. 2009;6(5): 1343-1352.
32. Kunnumakkara AB, Anand P, Aggarwal BB. Curcumin inhibits proliferation, invasion, angiogenesis and metastasis of different cancers through interaction with multiple cell signaling proteins. Cancer Lett. 2008;269(2):199-225.

33. Yallapu MM, Jaggi M, Chauhan SC. Curcumin nanomedicine: a road to cancer therapeutics. Curr Pharm Des. 2013;19(11):1994-2010.

34. Maheshwari RK, Singh AK, Gaddipati J, Srimal RC. Multiple biological activities of curcumin: a short review. Life Sci. 2006;78(18): 2081-2087.

35. Ranjan AP, Mukerjee A, Helson L, Gupta R, Vishwanatha JK. Efficacy of liposomal curcumin in a human pancreatic tumor xenograft model: inhibition of tumor growth and angiogenesis. Anticancer Res. 2013;33(9):3603-3609.

36. Sun J, Bi C, Chan HM, Sun S, Zhang Q, Zheng Y. Curcumin-loaded solid lipid nanoparticles have prolonged in vitro antitumour activity, cellular uptake and improved in vivo bioavailability. Colloids Surf B Biointerfaces. 2013;111C:367-375.

37. Altunbas A, Lee SJ, Rajasekaran SA, Schneider JP, Pochan DJ. Encapsulation of curcumin in self-assembling peptide hydrogels as injectable drug delivery vehicles. Biomaterials. 2011;32(25): 5906-5914.

38. Xu Q, Liu Y, Su S, et al. Anti-tumor activity of paclitaxel through dual-targeting carrier of cyclic RGD and transferring conjugated hyperbranched copolymer nanoparticles. Biomaterials. 2012;33(5): $1627-1639$

39. Su CL, Wang YT, Chang MH, Fang K, Chen K. The novel heterocyclic trioxirane [(1,3,5-tris oxiran-2-yl)methyl)-1,3,5-triazinane-2,4,6-trione (TATT)] exhibits a better anticancer effect than platinum-based chemotherapy by induction of apoptosis and curcumin further enhances its chemosensitivity. Cell Biochem Biophys. September 28, 2013. [Epub ahead of print.]

40. Gupta S, Jain A, Chakraborty M, Sahni JK, Ali J, Dang S. Oral delivery of therapeutic proteins and peptides: a review on recent developments Drug Deliv. 2013;20(6):237-246.

41. Bakhru SH, Furtado S, Morello AP, Mathiowitz E. Oral delivery of proteins by biodegradable nanoparticles. Adv Drug Deliv Rev. 2013;65(6):811-821.

42. Longmire M, Choyke PL, Kobayashi H. Clearance properties of nanosized particles and molecules as imaging agents: considerations and caveats. Nanomedicine (Lond). 2008;3(5):703-717. 


\section{Supplementary materials}

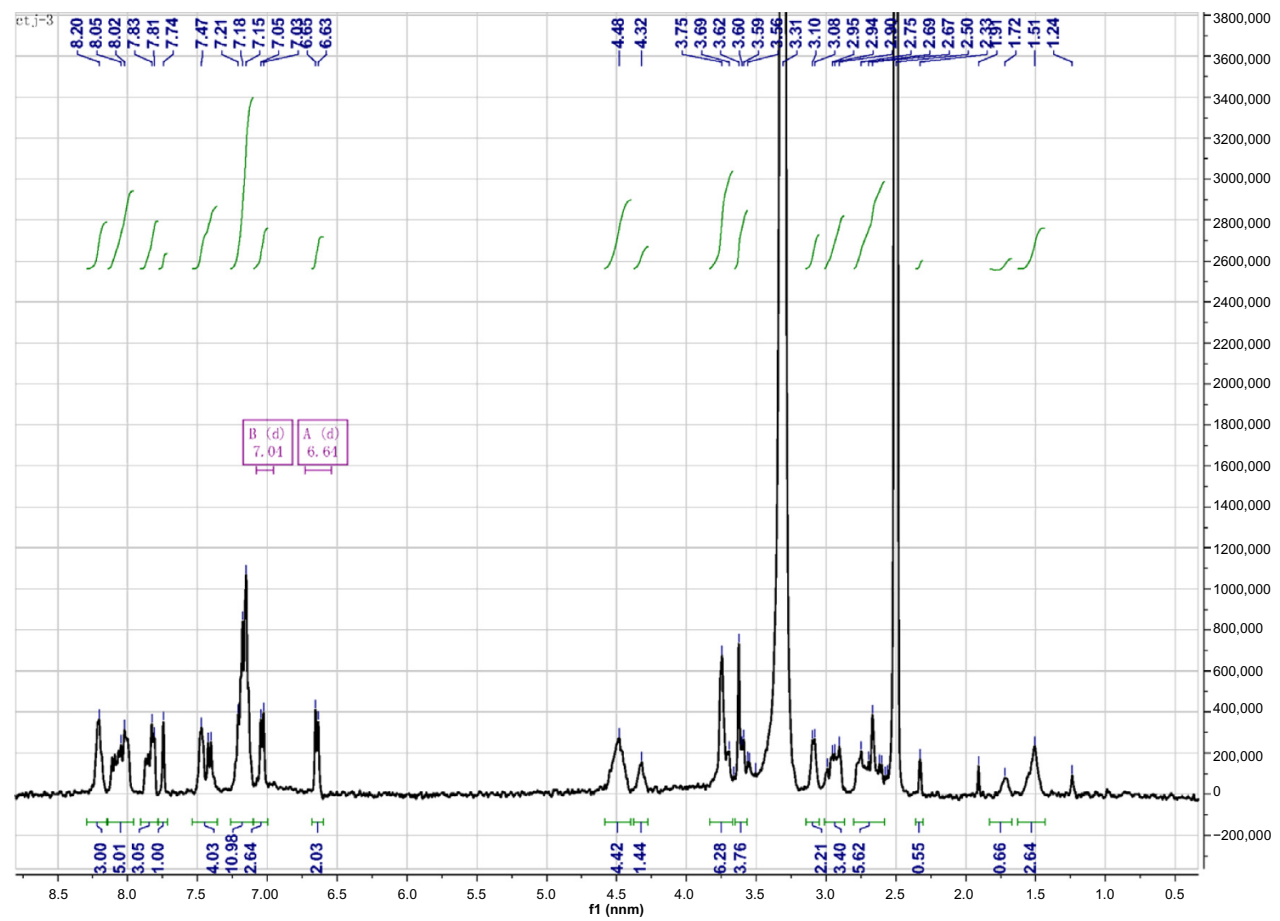

Figure SI 'H NMR spectrum of Nap-GFFYG-RGD.

Notes: Nap-GFFYG-RGD: 'H NMR (400 MHz, DMSO-d6) $\delta 8.20$ (s, 3H); 8.02-8.05 (m, 5H); 7.80-7.84 (m, 3H); $7.74(\mathrm{~s}, \mathrm{IH}) ; 7.40-7.47$ (m, 4H); 7.15-7.2I (m, IIH); 7.04 (d, 2H); $6.64(\mathrm{~d}, 2 \mathrm{H}) ; 4.40-4.49(\mathrm{~m}, 4 \mathrm{H}) ; 4.30-4.35(\mathrm{~m}, \mathrm{IH}) ; 3.69-3.78(\mathrm{~m}, 6 \mathrm{H}) ; 3.59-3.66(\mathrm{~m}, 4 \mathrm{H}) ; 3.08-3.10(\mathrm{~m}, 2 \mathrm{H}) ; 2.90-2.99(\mathrm{~m}, 3 \mathrm{H}) ; 2.56-2.75(\mathrm{~m}, 6 \mathrm{H}) ; 2.33(\mathrm{~s}, \mathrm{IH})$; I.72 (m, IH); I.50-I.53 (m, 3H). HR-MS: calc. $\mathrm{M}^{+}=1,085.4607$, obsvd $(\mathrm{M}+\mathrm{H})^{+}=1,086.4686$.

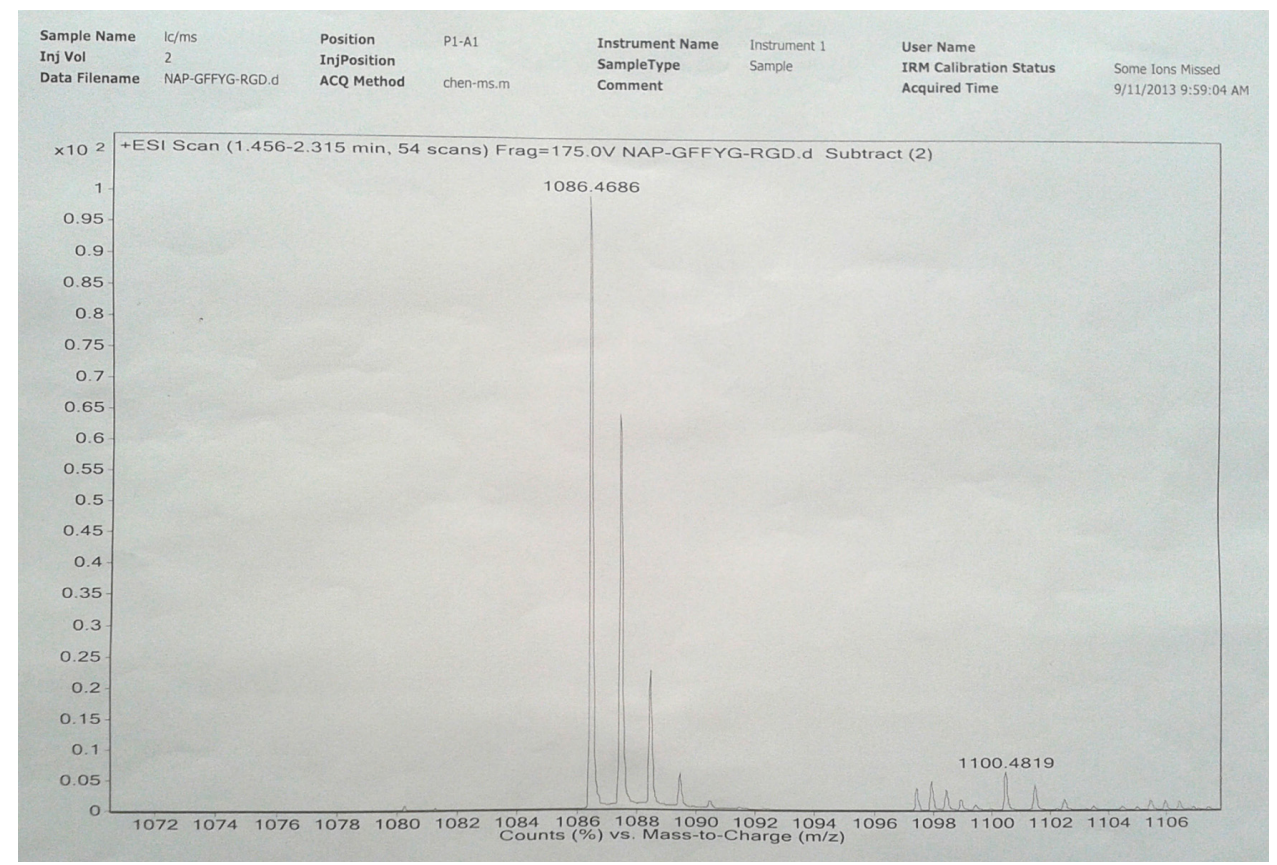

Figure S2 HR-MS of Nap-FFG.

Notes: Nap-GFFYG-RGE: 'H NMR (400 MHz, DMSO-d6) $\delta 8.22(\mathrm{~s}, 3 \mathrm{H}) ; 8.02-8.09(\mathrm{~m}, 6 \mathrm{H}) ; 7.83-7.85(\mathrm{~m}, 3 \mathrm{H}) ; 7.74(\mathrm{~s}, \mathrm{IH}) ; 7.40-7.47(\mathrm{~m}, 4 \mathrm{H}) ; 7.15-7.18(\mathrm{~m}, \mathrm{IIH})$; $7.03(\mathrm{~d}, 2 \mathrm{H}) ; 6.65(\mathrm{~d}, 2 \mathrm{H}) ; 4.35-4.50(\mathrm{~m}, 4 \mathrm{H}) ; 4.25-4.32(\mathrm{~m}, 4 \mathrm{H}) ; 3.70-3.76(\mathrm{~m}, 7 \mathrm{H}) ; 3.58-3.62(\mathrm{~m}, 8 \mathrm{H}) ; 3.09(\mathrm{~s}, 3 \mathrm{H}) ; 2.90-2.94(\mathrm{~m}, 4 \mathrm{H}) ; 2.65-2.78(\mathrm{~m}, 5 \mathrm{H}) ; 2.30-2.35(\mathrm{~m}, 3 \mathrm{H}) ;$ I.40-I.52 (m, 3H); I.243 (s, IH). HR-MS: calc. $M^{+}=I, 099.4763$, obsvd $(M+H)^{+}=I, 100.4829$. 


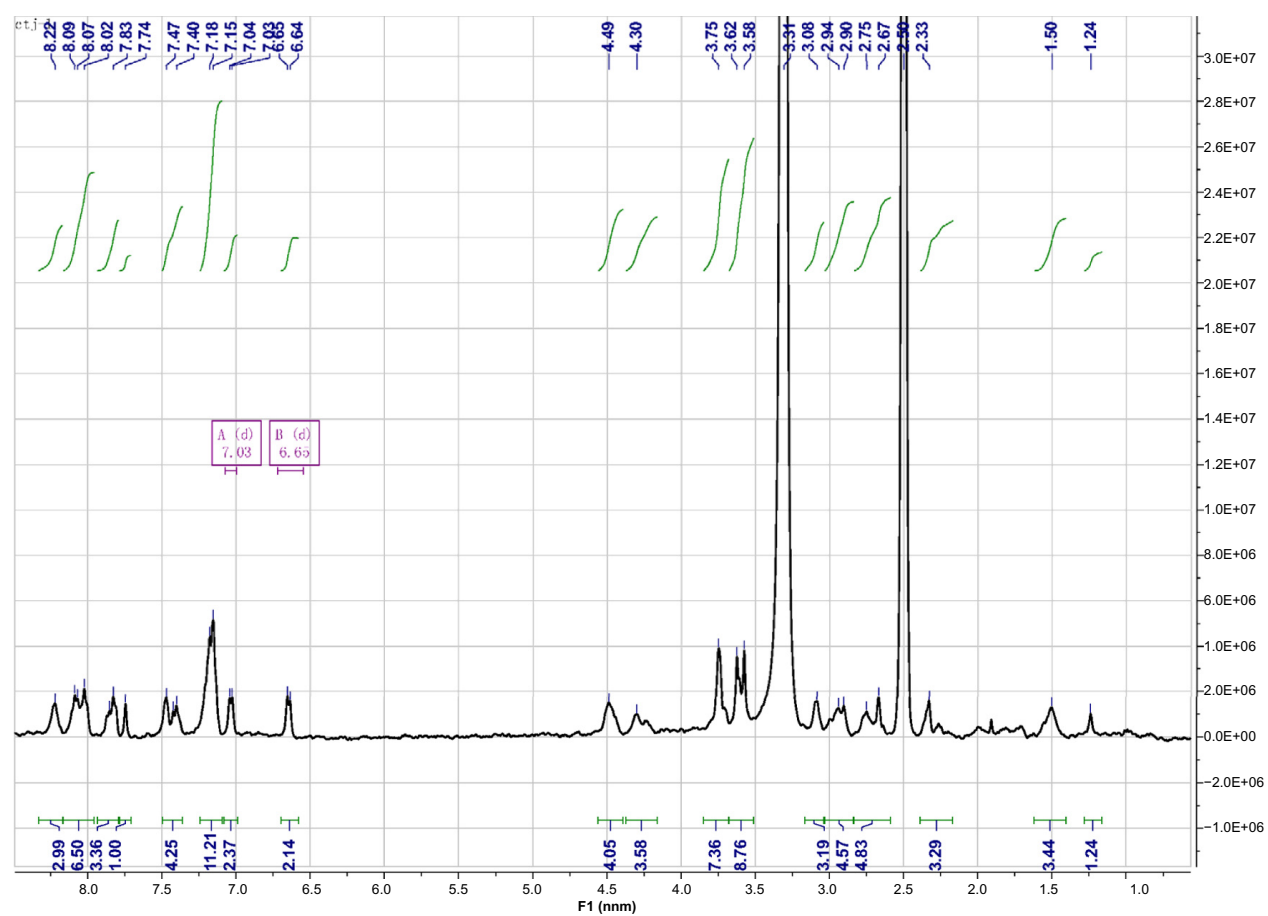

Figure S3 'H NMR spectrum of Nap-GFFYG-RGE.

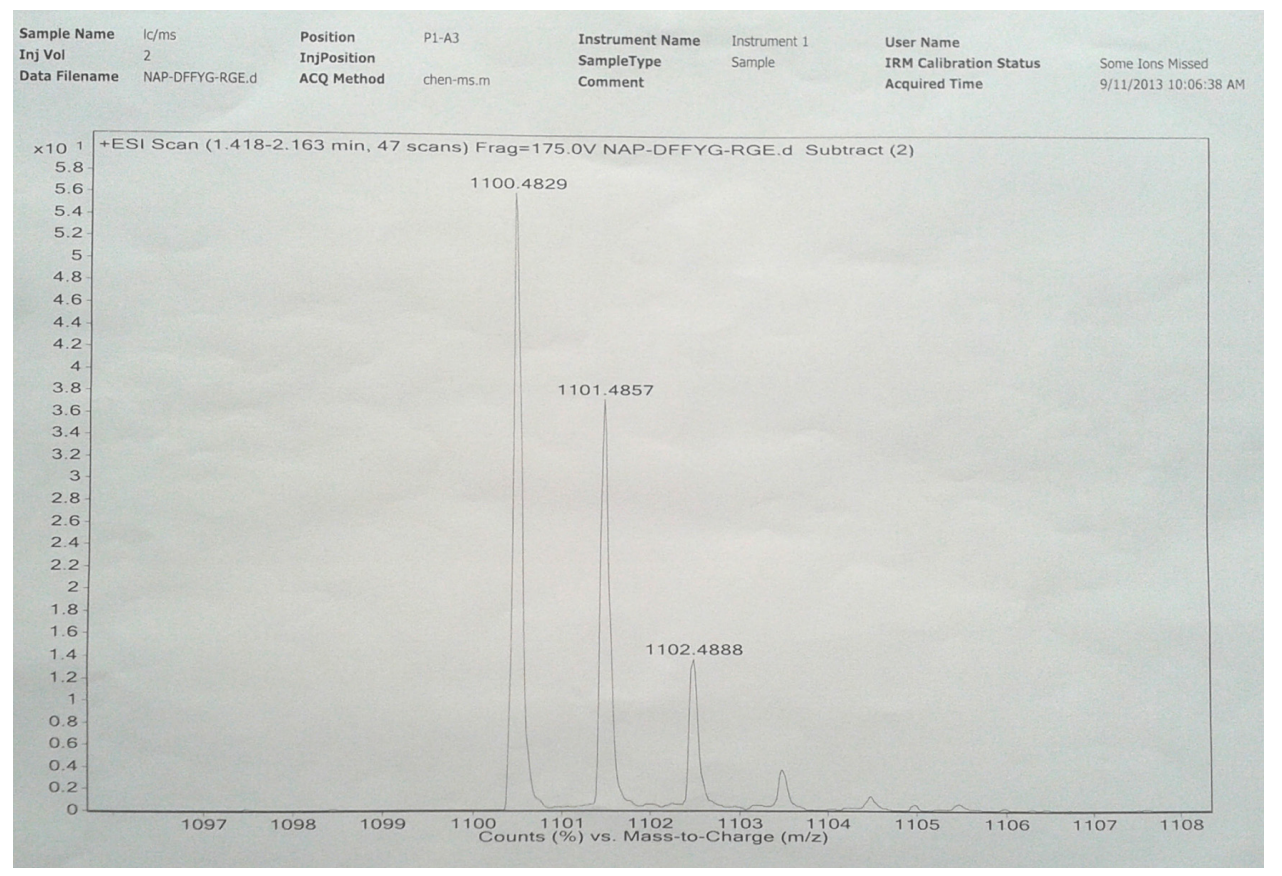

Figure S4 HR-MS of Nap-FFG.

International Journal of Nanomedicine

\section{Publish your work in this journal}

The International Journal of Nanomedicine is an international, peerreviewed journal focusing on the application of nanotechnology in diagnostics, therapeutics, and drug delivery systems throughou the biomedical field. This journal is indexed on PubMed Central, MedLine, CAS, SciSearch ${ }^{\circledR}$, Current Contents ${ }^{\circledR} /$ Clinical Medicine,

\section{Dovepress}

Journal Citation Reports/Science Edition, EMBase, Scopus and the Elsevier Bibliographic databases. The manuscript management system is completely online and includes a very quick and fair peer-review system, which is all easy to use. Visit http://www.dovepress.com/ testimonials.php to read real quotes from published authors.

Submit your manuscript here: http://www.dovepress.com/international-journal-of-nanomedicine-journal 